\title{
A Catalog of Ideas for Underground Yield Determination
}

\author{
L. F. Wouters
}

\section{DISCLAIMER}

This report was prepared as an account of work sponsored by an agency of the United States Government. Neither the United States Gnvernment nor any agency thereof, nor any of their employees, makes any warranty, express or implied, or assumes any legal liability or responsibility for the accuracy, completeness, or usefulness of any information, apparatus, product, or process disclosed, or represents that its use would not infringe privately owned rights. Reference herein to any specific commercial product, process, or service by trade name, trademark, manufacturer, or otherwise does not necessarily constitute or imply its endorsement, recommendation, or favoring by the United States Government or any agency thereof. The views and opinions of authors expressed herein do not necessarily state or reflect those of the United States Government or any agency thereof.

301010

JAN 301997

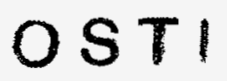

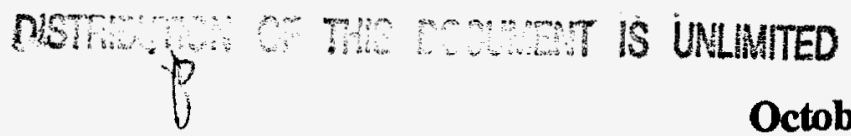

October 21, 1968

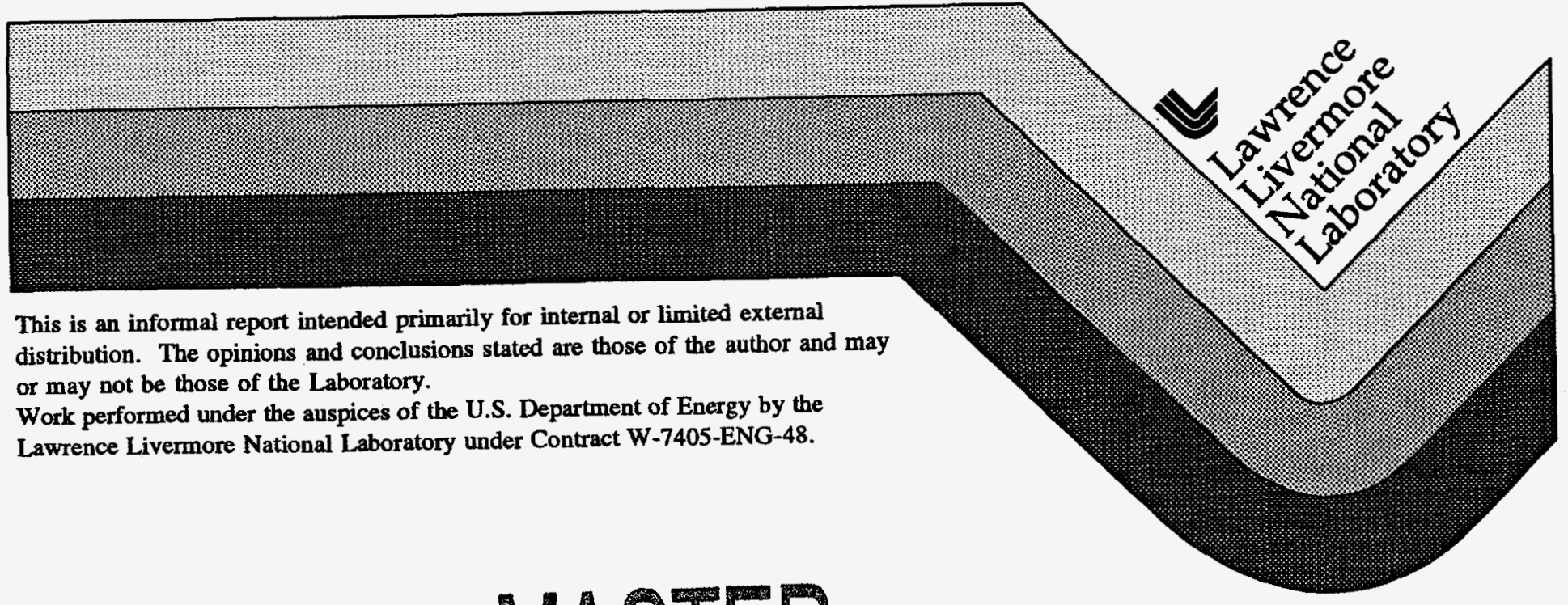




\section{DISCLAIMER}

Portions of this document may be illegible in electronic image products. Images are produced from the best available original document. 
Classification (Declassification/Review Date) Changed 10:

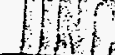

(Insegr appropriate elassification level or indicare Unclassificd

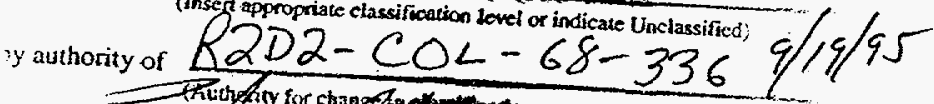

\section{$4^{2} / 183$}

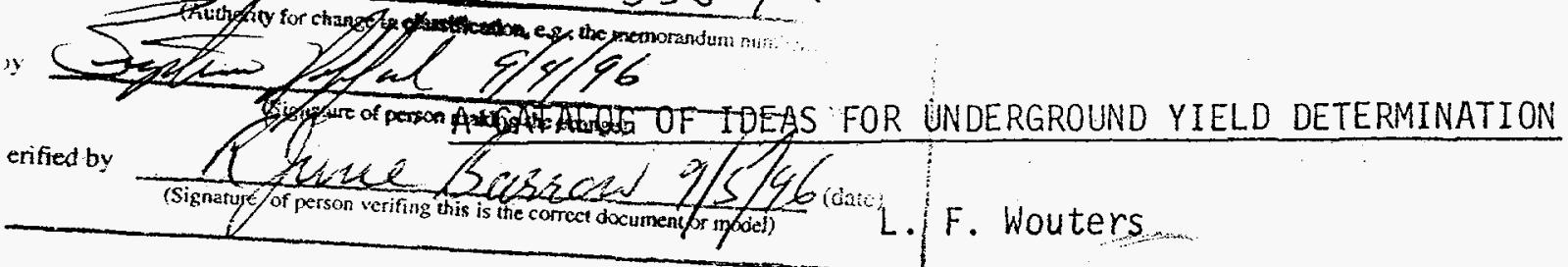

October 21, 1968

Because of the persistent problem of convenient determination of underground yield, I am summarizing certain "electromagnetically" oriented ideas in this area. They all require much more detailed study and evaluation, although one or two of them are inexpensive enough to perhaps warrant an empiric test.

[ 1. Vertical Sounding of the Ionosphere. The vertical displacement of the earth's surface (by the explosion) generates a sub-audible low-frequency acoustic wave which propagates approximately radially outward from surface zero. Subsequentiy, there appears to be a sufficient fluctuation in the ionospheric electron density to cause a detectable effect in grazing incidence reflection at H.F. (radio wavelengths)*. Vertical sounding by frequency-sweep methods usually provides a more sensitive measure of the electron density distribution perhaps sufficiently so to permit acoustic energy determination for events of nominal yield or larger.

This scheme would suffer generally from the same defects as direct motion measurements - namely, the coupled, acoustic energy will also

*The evidence for this will be communicated separately on reguest. 


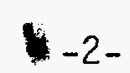

depend on burial depth, and on thie character and uniformity of the overburden. However, because the wave originates from the motion of a Targe surface area, the dispersion observed in surface accelerometer data (for instance) due to random differences along "ray paths" to the instrument positions, would tend to be "averaged out" - maybe.

Self-evidently, there would be considerable empiricism in such a scheme. But it is probably the simplest and easiest one to instrument. The equipment is well developed; it can be located several miles from surface zero; the measurement effort is readily purchasable as a package item. The experiment can be fielded and operated on a shorttime scale and on a minimum interference basis; there may not even be a "synchronization" probiem.

2. The EM "Magnetic Bubble" Scheme. This is the "original" underground EM generation mechanism first outlined by $0^{\prime}$ Rourke and this writer in 1960. It involves the interaction of the hot conducting gas plasma in the expanding cavity with the earth's magnetic field. One idea was that the amplitude and/or frequency distribution of the pulse, might be related to the cavity size. Extensive calculational work was sponsored by ARPA and DASA in the early. sixties (relative to underground test detection). The surface field predictions were discouragingly sma11, even "close-in", for a contained event.

In 1963 and 1964 , an extensive experimental program was conducted to try to identify this feature in the EM environment (a11 on cased events). It was found that the local magnetic field due to Compton currents on the casing was overwhelmingly large (by 2 to 3 orders of magnitude). No "late" signal component related to the orientation of 


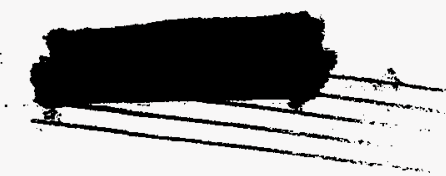

$-3-$

the earth's magnetic field was observed.

Incidentally, the calculations also indicated that the propagation attenuation is so high that, even without interference from other mechanisms and sources, the expected signal would fall below the ambient noise level in the earth's field for burial depths greater than about 800 feet in alluvial or other "wet" media.

3. "Non-Resonant Scattering". The earth's magnetic field is replaced by an artificially imposed field in this scheme. In particular, it would be a low-frequency field (order of 100 to $1000 \mathrm{cps}$ ). This offers an intrinsically higher ultimate signal-to-noise ratio, by virtue of narrow-band and phase-lock instrumentation techniques.

But it suffers from severe background problems, in that the receiver sensor $(R)$ must detect a degraded; re-radiated signal within the strong field of the transmitter $(T)$. Its degradation is a consequence of:

$$
\begin{aligned}
& \frac{e^{-a r}}{r} \text { propagation factor, transmitter to "bubble". } \\
& \text { Low re-radiation efficiency of "bubble" (10\% at best). } \\
& \frac{e^{-a r}}{r} \text { propagation factor, "bubble" to receiver. }
\end{aligned}
$$

The best geometry involves placing both the radiator and the sensor underground, with the "bubble" in between. Aside from cost, this has real problems in system survival through initial shock, inasmuch as one element (preferably the transmitter) should be "as close as possible" to the burst point.

Confining ourselves to near-surface configurations for both $T$ and $R$ elements, various arrangements were calculated. The required 


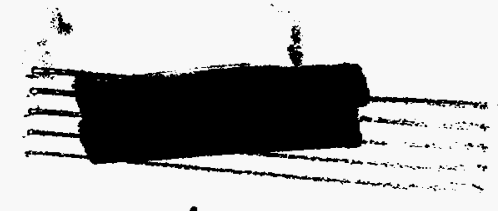

$-4-$

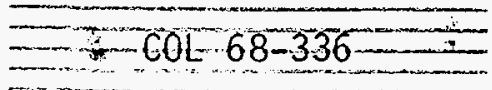

signal-to-background ratio is of the order of $10^{-9}$ to $10^{-10}$. The optimum arrangement (so far) appears to consist of a very large dipole transmitter 10op, axially concentric with the emplacement shaft, and a smaller quadrupole receiver loop, also axially concentric. While this appears at first glance to represent the worst possible background coupling situation, it also turns out to be the best possible source coupling geometry (as compared for instance to smaller, separated, and tilted loops placed on opposite sides of surface zero). By "idealized" assumptions of quadrupole balancing, shielding and residual phase cancelTation, one can achieve a realistic "paper" ratio of about $10^{-7}$ - which evidently isn't good enough.

I plan to "test" some further possible configurations, such as phased arrays of ferrite sensors, and the use of downhole T/R element locations. But I am not optimistic.

4. "Resonant Scattering". A conducting spherical body immersed in a dielectric, exhibits certain resonant electromagnetic modes for field distributions exterior to its surface. (I emphasize this because many people assume at first that I am speaking of the we11-known internal modes of a spherical cavity - NOT SO). The fundamental "wrap-around" mode has a wavelength (in vacuum) of $\lambda=7.3 \mathrm{a}$. In a dielectric of constant $k_{g}$, it would be $\frac{7.3 a}{\sqrt{k_{g}}}$ (to first order) where a is the radius. If we again consider the "conducting bubble" for "nominal" yields, we see that the end-point wavelength corresponds to frequencies in the low megacycles. This leads at once to the conclusion that one would

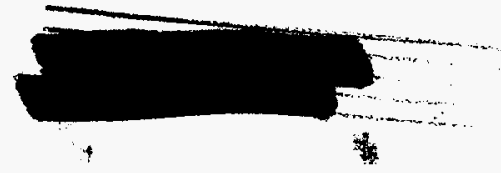




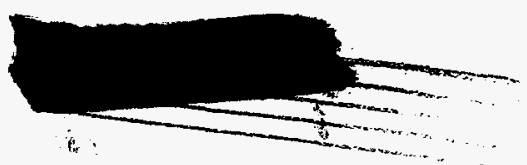

$-5-$

almost certainly not be able to observe this resonance in media of high conductivity such as tuff or alluvium. The test medium should have a resistivity greater than about 1000 ohm-meter.

This resonance is "strongly-coupled" - that is, one should see a uniquely high absorption cross-section at resonance (as compared to off-resonance - which actually corresponds to the previous non-resonant scheme). Stratton shows that the radiation damping is so great, that the resonance energy falls by $e^{-1}$ in one-quarter cycle!

There appear to be many ways to "see" this resonance if one is in a reasonably transparent medium. The great advantage of a resonance technique, is of course, that the determination of radius, cavity volume, and (hopefully) yjeld, now becomes related to a frequency measurement, rather than amplitude. What's more, some of the techniques may permit determination of cavity growth as a function of time (beyond about .3 millisec.), which would provide a firmer handle on yield.

Most of the schemes involve illumination by one or more transmitters, and observation by one or more receivers. Briefly, they sort out as follows:

Set of fixed frequency $T \& R$ - observe time of appearance of each resonance - use phase lock to upgrade sensitivity if necessary. Frequency sweep $T \& R$ (essentially similar). Puised $T \& R$ adjusted to examine scattered signal between $T$ putses.

Doppler-shift receiver to observe frequency dispersion due to expansion velocity of cavity surface.

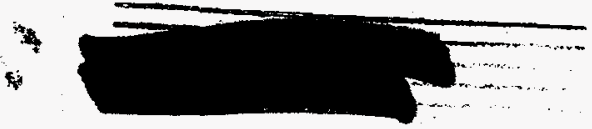


Several interesting radiator-sensor geometries have been proposed, which take advantage of unique features of resonant scattering. For instance: $T$ below test capsule (at bottom of over-drilled hole), $R$ at top of hole (or several sensors along the hole) - essentialiy this is a "good geometry" diffraction experiment. At particular frequencies, minima and maxima in signal ampiitude should be seen, as the points of maximum constructive and destructive interference move outward along the axis, during cavity growth.

One other possible and unusual coupling scheme makes use of a single electronic device - a conditionally stable, broad-band, positive feedback amplifier, connected to an "appropriate sensor" (or array). It would be designed and adjusted to be stable in the absence of resonant elements in its field. When such an element appears, the feedback phase shifts enough to throw the system into oscillation - and it automatically "locks on" to the resonant frequency. In this scheme, it would then "follow" the cavity growth. Pulse "tick7ing" might be needed.

Detailed calculation of the field coupling features are under "hot pursuit".

5. The Gravitational Pulse. (This one is really far out). Instrumentation for measuring gravitational fluctuations has improved greatly, along with an understanding of the effect of such a pulse in the laboratory frame.

Two mechanisms may exist in underground nuclear explosions leading to generation of gravitational pulses. One of these is simply the 


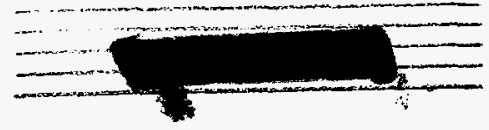

conversion of mass to energy by the nuclear reactions. In a primitive model, one would say that the mass of the reactants is changed by $\Delta m=\frac{Y}{c^{2}}$, on the time scale of reaction (7ike $\mathrm{T} \sim 10^{-8} \mathrm{sec}$. for modern devices). But it is not clear that the effective mass disappears that rapidiy from the gravitational interaction. Nevertheless, proceeding on this primitive assumption, one obtains a rate of mass destruction of $\sim 10^{4} \mathrm{~kg} / \mathrm{sec}$ per $\mathrm{kt}$. The technique used for observation of galactic pulses (presumed due to imploding neutron stars) has a rate sensitivity, which translated to terrestrial dimensions; corresponds to about $10^{4}$ $\mathrm{kg} / \mathrm{sec}$ at $1 \mathrm{~km}$. Thus, it is not too incredible to take a hard look at this - besides, it is intensely fascinating!

This "prompt" pulse has a wavelength cr short compared to the experimental dimensions. It is thus (presumably) a tensor field pulse, which has the interesting property of "squeezing" a mass rather than displacing it. The tensor (or squeeze) direction is orthogonal to the direction of energy propagation, analogous to an EM field vector. Since that field acts individuaily on the molecules, the sensor object undergoes a mechanical shock essentialiy "simultaneously" (on a $T$ time scale) throughout its volume. A primitive sensor concept might be a $U^{23}$ bar about 10 meters long (and $1 \mathrm{~m}^{2}$ cross-section:), whose length would be measured by means of a Laser driven interferometer. It looks like one might do this to about one part in $10^{15}$, even for a mechanical pulse this short. (This is also the order of sensitivity of modern gravitational sensors). It's been suggested that one might go downhole with such a device to gain in coupling:

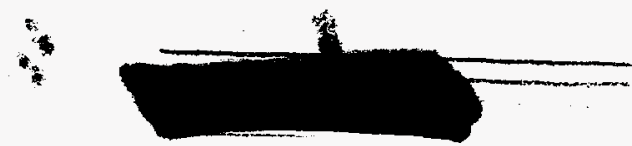


The other possible mechanism is related to the change in the (mechanical) moment of the local gravitational field when the cavity expands. At first glance, one would say that, since the center of mass does not shift (in an idealized spherical explosion), there should be no change in the external field. (Sort of like a gravitational Faraday cage).

This "slow" pulse has an enormous wavelength, of course, since the mass reconfiguration occurs on a very slow time scale. Hence, it is a quasi-static situation and the field pulse is a radially scalar one, describable by the classical gravitational equation in integral form:

$$
\Delta F=G M \int_{2}\left(\frac{\rho}{r^{2}}\right) d v-G M \int_{1}\left(\frac{\rho}{r^{2}}\right) d v
$$

This "after-before" integral appears to have a sma11, non-zero residue which is independent of the method of (arithmetic) expansion. Evidently further analysis is needed to understand this one.

If it is real, this field fluctuation should longitudinally displace a sensor mass in the classical way. One would expect a frequency spectrum centered at: something like the cavity expansion rate - i.e., $f \sim \frac{V}{r}$ or something around $100 \mathrm{cps}$. Accordingly, a sensor might be designed for peak sensitivity in this range, probably by the same mechanical principles as are employed in long-wave seismic instrumentation.

That's all for now.

LFW: Ian

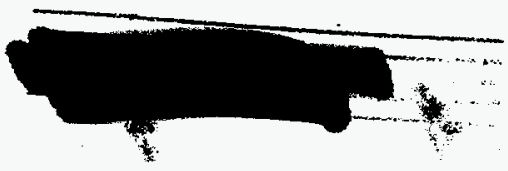




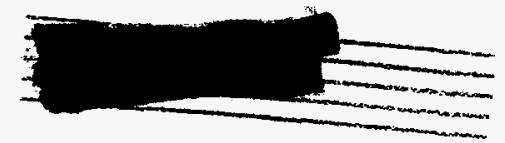

$-9-$

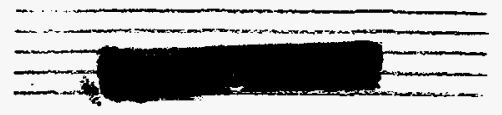

Distribution:

$\begin{array}{lll}1 \text { of } 14 \mathrm{~A} & \mathrm{~J} \text {. Brittingham } \\ 2 \text { of } 14 \mathrm{~A} & \mathrm{~J} \text {. Carothers } \\ 3 & \text { of } 14 \mathrm{~A} & \mathrm{R} \text {. Gathers } \\ 4 \text { of } 14 \mathrm{~A} & \mathrm{~L} \text {. Germain } \\ 5 & \text { of } 14 \mathrm{~A} & \mathrm{~F} \text {. Gilbert } \\ 6 & \text { of } 14 \mathrm{~A} & \mathrm{G} \text {. Higgins } \\ 7 \text { of } 14 \mathrm{~A} & \mathrm{C} \text {. Maninger } \\ 8 \text { of } 14 \mathrm{~A} & \mathrm{~W} \text {. McMaster } / \mathrm{V} \text {. Wheeler } \\ 9 \text { of } 14 \mathrm{~A} & \mathrm{H} \text {. Reynolds } \\ 10 \text { of } 14 \mathrm{~A} & \mathrm{~J} \text {. Rosengren/M. Knapp } \\ 11 \text { of } 14 \mathrm{~A} & \mathrm{~J} \text {. Watson } \\ 12 \text { of } 14 \mathrm{~A} & \mathrm{~L} \text {. Wouters } \\ 13 \text { of } 14 \mathrm{~A} & \mathrm{File} \\ 14 \text { of } 14 \mathrm{~A} & \mathrm{File}\end{array}$

